\title{
A STABILITY RESULT ON MUCKENHOUPT'S WEIGHTS
}

\author{
JuHA Kinnunen
}

\begin{abstract}
We prove that Muckenhoupt's $\mathcal{A}_{1}$-weights satisfy a reverse Hölder inequality with an explicit and asymptotically sharp estimate for the exponent. As a by-product we get a new characterization of $\mathcal{A}_{1}$-weights.
\end{abstract}

\section{Introduction and statement of results}

Muckenhoupt's weights are important tools in harmonic analysis, partial differential equations and quasiconformal mappings. The selfimproving property of Muckenhoupt's weights is probably one of the most useful results in the field. The surprising fact that the weights are more regular than they seem to be a priori was observed already by Muckenhoupt [16]. The same phenomenon was studied by Gehring in [6] where he introduced the concept of reverse Hölder inequalites and proved that they improve themselves. Later Coifman and Fefferman [3] showed that Muckenhoupt's weights are exactly those weights which satisfy a reverse Hölder inequality. Since then reverse Hölder inequalities have had a vast number of applications in modern analysis. An excellent source for all the mentioned results and other properties of Muckenhoupt's weights is the monograph [7].

We are interested in a stability question related to Muckenhoupt's $\mathcal{A}_{1}$-class and reverse Hölder inequalities. Suppose that $w: \mathbf{R}^{n} \rightarrow[0, \infty]$ is a locally integrable function satisfying Muckenhoupt's $\mathcal{A}_{1}$-condition,

$$
\frac{1}{|B|} \int_{B} w(x) d x \leq c_{w} \underset{x \in B}{\operatorname{essinf}} w(x)
$$

Keywords. Muckenhoupt weight, reverse Hölder inequality. 1991 Mathematics subject classifications: 42B26. 
for all balls $B \subset \mathbf{R}^{n}$ with the constant $c_{w} \geq 1$ independent of the ball $B$. Here $|B|$ is the volume of $B$. If $w$ belongs to Muckenhoupt's class $\mathcal{A}_{1}$, we denote $w \in \mathcal{A}_{1}$; the smallest constant $c_{w}$ for which (1.1) holds is called the $\mathcal{A}_{1}$-constant of $w$.

Condition (1.1) can be expressed in terms of the Hardy-Littlewood maximal function, defined by

$$
\mathcal{M} w(x)=\sup _{B} \frac{1}{|B|} \int_{B} w(y) d y
$$

where the supremum is over all balls $B \subset \mathbf{R}^{n}$ containing the point $x$. It is easy to see $[\mathbf{7}$, p. 389] that (1.1) is equivalent to the requirement that

$$
\mathcal{M} w(x) \leq c_{w} w(x)
$$

almost everywhere with exactly the same $c_{w}$ as in (1.1).

It is clear that (1.1) imposes a serious restriction on the function. If the $\mathcal{A}_{1}$-constant is one, then

$$
0 \leq \frac{1}{|B|} \int_{B}(w(y)-\underset{x \in B}{\operatorname{essinf}} w(x)) d y \leq \underset{x \in B}{\operatorname{essinf}} w(x)-\underset{x \in B}{\operatorname{essinf}} w(x)=0
$$

and hence $w$ is constant. We are interested in the regularity of $\mathcal{A}_{1}$-weights as the constant tends to one. It is well-known that $\mathcal{A}_{1}$-weights satisfy the reverse Hölder inequality

$$
\left(\frac{1}{|B|} \int_{B} w(x)^{p} d x\right)^{1 / p} \leq c \frac{1}{|B|} \int_{B} w(x) d x
$$

for some $p>1$ and $c$ independent of the ball $B$. Using (1.2) and (1.1) we see that $w^{p} \in \mathcal{A}_{1}$ and $w$ is locally integrable to power $p$. The question is: how large can $p$ be? If the $\mathcal{A}_{1}$-constant is one, then the weight is essentially bounded and it seems reasonable to expect that the degree of the local integrablity increases as the $\mathcal{A}_{1}$-constant tends to one. Questions related to the stability of reverse Hölder inequalities have obtained considerable attention in the last two decades, see $[\mathbf{1}],[\mathbf{2}],[\mathbf{9}],[\mathbf{1 1}],[\mathbf{1 2}]$, $[\mathbf{1 3}],[\mathbf{1 4}],[\mathbf{1 5}],[\mathbf{1 7}],[\mathbf{1 8}],[\mathbf{1 9}],[\mathbf{2 0}]$ and $[\mathbf{2 1}]$.

Our contribution is twofold. First, we present a new and a simple method which gives an explicit and asymptotically optimal bound for $p$. Second, our proof leads to a new characterization of $\mathcal{A}_{1}$-weights (Corollary 2.11) which may be of independent interest.

Now we are ready to present our main result. 
1.3. Theorem. If $w \in \mathcal{A}_{1}$ with the constant $c_{w}$, then there is a constant $\nu$ depending only on the dimension such that $w$ satisfies the reverse Hölder inequality (1.2) whenever

$$
1 \leq p<1+\frac{\nu}{c_{w}-1} .
$$

In the one-dimensional case we may take $\nu=1$ in (1.4), see [2] and [11], but our proof generally yields a small $\nu$. Our method also allows us to replace balls in the $\mathcal{A}_{1}$-condition by cubes. Observe that the bound (1.4) for the local integrability of the weight is arbitrarily large provided $c_{w}$ is close enough to one.

We remark that using factorization results of [10] and [4], our method gives similar estimates for Muckenhoupt's $\mathcal{A}_{p}$-weights as well. In the one-dimensional case this has been studied by Neugebauer [17].

\section{Characterization of $\mathcal{A}_{1}$-weights}

We begin by showing that every $\mathcal{A}_{1}$-weight can be approximated by smooth $\mathcal{A}_{1}$-weights.

2.1. Lemma. Suppose that $w \in \mathcal{A}_{1}$ with the constant $c_{w}$ and let $\varphi \in C_{0}^{\infty}\left(\mathbf{R}^{n}\right), \varphi \geq 0$ with $\int_{\mathbf{R}^{n}} \varphi d x=1$. Then $w * \varphi \in \mathcal{A}_{1}$ with the constant $c_{w}$.

Proof: A direct calculation gives

$$
\begin{aligned}
\frac{1}{B(x, r)} \int_{B(x, r)} w * \varphi(y) d y & =\frac{1}{B(x, r)} \int_{B(x, r)} \int_{\mathbf{R}^{n}} w(y-z) \varphi(z) d z d y \\
& =\int_{\mathbf{R}^{n}} \varphi(z) \frac{1}{B(x-z, r)} \int_{B(x-z, r)} w(y) d y d z \\
& \leq c_{w} \int_{\mathbf{R}^{n}} \varphi(z) \underset{y \in B(x-z, r)}{\operatorname{essinf}} w(y) d z \\
& =c_{w} \int_{\mathbf{R}^{n}} \varphi(z) \underset{y \in B(x, r)}{\operatorname{essinf}} w(y-z) d z \\
& \leq c_{w} \underset{y \in B(x, r)}{\operatorname{essinf}} \int_{\mathbf{R}^{n}} w(y-z) \varphi(z) d z \\
& =c_{w} \underset{y \in B(x, r)}{\operatorname{essinf}} w * \varphi(y) .
\end{aligned}
$$

This completes the proof.

We record a well-known covering theorem. 
2.3. Besicovitch's covering Theorem. Suppose that $E$ is a bounded subset of $\mathbf{R}^{n}$ and that $\mathcal{B}$ is a collection of balls such that each point of $E$ is a center of some ball in $\mathcal{B}$. Then there exists an integer $N \geq 2$ (depending only on the dimension) and subcollections $\mathcal{B}_{1}, \ldots, \mathcal{B}_{N} \subset \mathcal{B}$ of at most countably many balls such that the balls $B_{i, j}$, $j=1,2, \ldots$, in each family $\mathcal{B}_{i}, i=1,2, \ldots, N$, are pairwise disjoint and

$$
E \subset \bigcup_{i=1}^{N} \bigcup_{j=1}^{\infty} B_{i, j}
$$

For the proof of Besicovitch's covering Theorem we refer to [5, Theorem 1.1]. Some estimates for the constant $N$ are obtained in [8].

Now we show that $\mathcal{A}_{1}$-weights satisfy a reverse Chebyshev inequality. This observation is a crucial ingredient in the proof of Theorem 1.3. For short we denote

$$
E_{\lambda}=\left\{x \in \mathbf{R}^{n}: w(x)>\lambda\right\}, \quad \lambda>0,
$$

throughout the paper.

2.4. Lemma. Let $B \subset \mathbf{R}^{n}$ be a ball and suppose that $w: \mathbf{R}^{n} \rightarrow$ $[0, \infty]$ is an $\mathcal{A}_{1}$-weight with the constant $c_{w}$. Then there is a constant $\eta$, depending only on the dimension, so that

$$
\int_{E_{\lambda} \cap B} w(x) d x \leq\left(c_{w}+\eta\left(c_{w}-1\right)\right) \lambda\left|E_{\lambda} \cap B\right|,
$$

whenever $\operatorname{ess}_{\inf } \in B(x) \leq \lambda<\infty$.

Proof: Fix a ball $B \subset \mathbf{R}^{n}$. Suppose first that $w$ is a continuous $\mathcal{A}_{1}$-weight with the constant $c_{w}$ and that $\lambda \geq \inf _{x \in B} w(x)$. Then $E_{\lambda}$ is open and for every $x \in E_{\lambda}$ we take the ball $B\left(x, r_{x}\right)$ where $r_{x}$ is the distance from $x$ to the boundary of $E_{\lambda}$. Let $\mathcal{B}=\left\{B\left(x, r_{x}\right): x \in E_{\lambda} \cap B\right\}$. The radii of the balls in $\mathcal{B}$ are bounded, because $\bar{B} \backslash E_{\lambda} \neq \emptyset$. By Besicovitch's covering Theorem, there are families $\mathcal{B}_{i}=\left\{B_{i, j}: j=1,2, \ldots\right\}$, $i=1,2, \ldots, N$, of countably many balls, chosen from $\mathcal{B}$, such that

$$
E_{\lambda} \cap B=\bigcup_{i=1}^{N} \bigcup_{j=1}^{\infty} B_{i, j} \cap B
$$

and the balls in every $\mathcal{B}_{i}, i=1,2, \ldots, N$, are pairwise disjoint. We denote the union of the pairwise disjoint balls by

$$
E_{\lambda}^{i}=\bigcup_{j=1}^{\infty} B_{i, j}, \quad i=1,2, \ldots, N
$$


The balls $B_{i, j}$ touch the boundary of $E_{\lambda}$ and, since $w$ is continuous, using the $\mathcal{A}_{1}$-condition we get

$$
\begin{aligned}
\frac{1}{\left|B_{i, j}\right|} \int_{B_{i, j}} w(x) d x \leq c \inf _{x \in B_{i, j}} w(x) & \leq c \lambda \\
& i=1,2, \ldots, N, j=1,2, \ldots
\end{aligned}
$$

The balls $B_{i, j}$ are not, in general, contained in $B$, but there is a constant $\gamma>0$, depending only on the dimension, so that

$$
\left|B_{i, j} \backslash B\right| \leq \gamma\left|B_{i, j} \cap B\right|, \quad i=1,2, \ldots, N, j=1,2, \ldots
$$

To see this, let $B_{i, j}$ be the ball $B\left(x, r_{x}\right) \subset E_{\lambda}$ with $x \in E_{\lambda} \cap B$. Then by geometry, there is a ball $B\left(y, r_{x} / 2\right) \subset B\left(x, r_{x}\right) \cap B$. This gives us the estimate

$$
\left|B\left(x, r_{x}\right) \backslash B\right| \leq\left|B\left(x, r_{x}\right)\right|=2^{n}\left|B\left(y, r_{x} / 2\right)\right| \leq 2^{n}\left|B\left(x, r_{x}\right) \cap B\right| .
$$

Hence we may take $\gamma=2^{n}$.

By observing that $w(x)>\lambda$ for every $x \in B_{i, j}$ and recalling (2.6) we see that

$$
\begin{aligned}
\int_{B_{i, j} \cap B} w(x) d x & \leq c_{w} \lambda\left|B_{i, j} \cap B\right|+c_{w} \lambda\left|B_{i, j} \backslash B\right|-\int_{B_{i, j} \backslash B} w(x) d x \\
& \leq c_{w} \lambda\left|B_{i, j} \cap B\right|+\left(c_{w}-1\right) \lambda\left|B_{i, j} \backslash B\right| \\
& \leq\left(c_{w}+\gamma\left(c_{w}-1\right)\right) \lambda\left|B_{i, j} \cap B\right|, \\
& \quad i=1,2, \ldots, N, j=1,2, \ldots
\end{aligned}
$$

Since the balls in each $\mathcal{B}_{i}, i=1,2, \ldots, N$, are pairwise disjoint, we arrive at

$$
\begin{aligned}
\int_{E_{\lambda}^{i} \cap B} w(x) d x & =\sum_{j=1}^{\infty} \int_{B_{i, j} \cap B} w(x) d x \\
& \leq\left(c_{w}+\gamma\left(c_{w}-1\right)\right) \lambda\left|E_{\lambda}^{i} \cap B\right|, \quad i=1,2, \ldots, N
\end{aligned}
$$

Let $\mu$ be a measure. Then we use the elementary inequality

$$
\mu\left(E_{\lambda} \cap B\right)=\sum_{i=1}^{N} \mu\left(E_{\lambda}^{i} \cap B\right)-\sum_{k=2}^{N} \mu\left(F_{\lambda}^{k} \cap B\right),
$$


where

$$
F_{\lambda}^{k}=\bigcup_{\left\{l_{1}, \ldots, l_{k}\right\} \subset\{1, \ldots, N\}}\left(E_{\lambda}^{l_{1}} \cap \cdots \cap E_{\lambda}^{l_{k}}\right), \quad k=2,3, \ldots, N .
$$

A simple computation using (2.8), (2.7) and the fact that $w(x)>\lambda$ in $F_{\lambda}^{k} \cap B, k=2, \ldots, N$, gives

$$
\begin{array}{rl}
\int_{E_{\lambda} \cap B} & w(x) d x=\sum_{i=1}^{N} \int_{E_{\lambda}^{i} \cap B} w(x) d x-\sum_{k=2}^{N} \int_{F_{\lambda}^{k} \cap B} w(x) d x \\
& \leq\left(c_{w}+\gamma\left(c_{w}-1\right)\right) \lambda \sum_{i=1}^{N}\left|E_{\lambda}^{i} \cap B\right|-\lambda \sum_{k=2}^{N}\left|F_{\lambda}^{k} \cap B\right| \\
& =\left(c_{w}+\gamma\left(c_{w}-1\right)\right) \lambda\left|E_{\lambda} \cap B\right|+\lambda(1+\gamma)\left(c_{w}-1\right) \sum_{k=2}^{N}\left|F_{\lambda}^{k} \cap B\right| \\
& \leq\left(c_{w}+\gamma\left(c_{w}-1\right)\right) \lambda\left|E_{\lambda} \cap B\right|+(N-1)(1+\gamma)\left(c_{w}-1\right) \lambda\left|E_{\lambda} \cap B\right| \\
& =\left(c_{w}+\eta\left(c_{w}-1\right)\right) \lambda\left|E_{\lambda} \cap B\right|,
\end{array}
$$

where $\eta=N \gamma+N-1$ and $\lambda \geq \inf _{x \in B} w(x)$.

The general case follows from a standard approximation argument using Lemma 2.1. Suppose that $w \in \mathcal{A}_{1}$ with the constant $c_{w}$. Let $\varphi \in C_{0}^{\infty}\left(\mathbf{R}^{n}\right), \varphi \geq 0$ with $\int_{\mathbf{R}^{n}} \varphi d x=1$. We define $w_{\varepsilon}=w * \varphi_{\varepsilon}$, where $\varphi_{\varepsilon}(x)=\varepsilon^{-n} \varphi(x / \varepsilon)$ and $\varepsilon>0$. Lemma 2.1 shows that $w_{\varepsilon}$ is a continuous $\mathcal{A}_{1}$-weight with the constant $c_{w}$ for every $\varepsilon>0$. Using (2.9) we see that

$$
\begin{aligned}
& \int_{\left\{w_{\varepsilon}>\lambda\right\} \cap B} w_{\varepsilon}(x) d x \leq\left(c_{w}+\eta\left(c_{w}-1\right)\right) \lambda\left|\left\{w_{\varepsilon}>\lambda\right\} \cap B\right|, \\
& \inf _{x \in B} w_{\varepsilon}(x) \leq \lambda<\infty .
\end{aligned}
$$

Letting $\varepsilon \rightarrow 0$ we obtain (2.5). This completes the proof.

2.10. Remark. (1) Observe that the constant on the right side of (2.5) tends to one as $c_{w}$ tends to one. On the other hand, it blows up as $c_{w}$ increases.

(2) We also remark that inequalities of type (2.5) appear already in the proof of Theorem 4 in $[\mathbf{3}]$. However, their approach does not seem to give the correct behaviour as $c_{w}$ tends to one.

We observe that (2.5) gives a characterization of $\mathcal{A}_{1}$-weights. 
2.11. Corollary. Suppose that $w: \mathbf{R}^{n} \rightarrow[0, \infty]$ is a measurable function. Then $w \in \mathcal{A}_{1}$ if and only if there is a constant $c$, independent of the ball $B$, so that

$$
\int_{E_{\lambda} \cap B} w(x) d x \leq c \lambda\left|E_{\lambda} \cap B\right|, \quad \underset{x \in B}{\operatorname{essinf}} w(x) \leq \lambda<\infty,
$$

for every ball $B \subset \mathbf{R}^{n}$.

Proof: Lemma 2.4 shows that every $\mathcal{A}_{1}$-weight satisfies (2.12).

To see the reverse implication suppose that (2.12) holds and let $B$ be a ball in $\mathbf{R}^{n}$. Then

$$
\begin{aligned}
\int_{B} w(x) d x & =\int_{B \backslash E_{\lambda}} w(x) d x+\int_{E_{\lambda} \cap B} w(x) d x \\
& \leq \lambda\left|B \backslash E_{\lambda}\right|+c \lambda\left|B \cap E_{\lambda}\right| \\
& \leq c \lambda|B|, \quad \underset{x \in B}{\operatorname{essinf}} w(x) \leq \lambda<\infty .
\end{aligned}
$$

By inserting $\lambda=\operatorname{essinf}_{x \in B} w(x)$ we get

$$
\frac{1}{|B|} \int_{B} w(x) d x \leq \underset{x \in B}{\operatorname{essinf}} w(x),
$$

where the constant is independent of the ball and hence $w \in \mathcal{A}_{1}$.

2.13. Remark. In the one-dimensional case we may take the constant in (2.12) equal to the $\mathcal{A}_{1}$-constant of $w$, see [11].

Lemma 2.4 shows that $w$ satisfies the assumptions of the following sharp version Muckenhoupt's Lemma 4 in [16]. See also Lemma 2 in [2]. The proof of the following lemma can be found in [11], but we present it here for the sake of completeness.

2.14. Lemma. Suppose that $w: \mathbf{R}^{n} \rightarrow[0, \infty]$ is a measurable function and let $B \subset \mathbf{R}^{n}$ be a ball. If there are $\alpha \geq 0$ and $c>1$ such that

$$
\int_{E_{\lambda} \cap B} w(x) d x \leq c \lambda\left|E_{\lambda} \cap B\right|, \quad \alpha \leq \lambda<\infty,
$$

then for every $p, 1<p<c /(c-1)$, we have

$$
\int_{E_{\alpha} \cap B} w(x)^{p} d x \leq \frac{c}{c-p(c-1)} \alpha^{p}\left|E_{\alpha} \cap B\right| .
$$


Proof: Let $\beta>\alpha$ and denote $w_{\beta}=\min (w, \beta)$. Then

$$
\int_{\left\{w_{\beta}>\lambda\right\} \cap B} w(x) d x \leq c \lambda\left|\left\{w_{\beta}>\lambda\right\} \cap B\right|, \quad \alpha \leq \lambda<\infty .
$$

We multiply both sides by $\lambda^{p-2}$ and integrate from $\alpha$ to $\infty$. This implies

$$
\int_{\alpha}^{\infty} \lambda^{p-2} \int_{\left\{w_{\beta}>\lambda\right\} \cap B} w(x) d x d \lambda \leq c \int_{\alpha}^{\infty} \lambda^{p-1}\left|\left\{w_{\beta}>\lambda\right\} \cap B\right| d \lambda .
$$

Then we use the equality

$$
\int_{E_{\alpha} \cap B} w(x)^{p} d \mu=p \int_{\alpha}^{\infty} \lambda^{p-1} \mu\left(E_{\lambda} \cap B\right) d \lambda+\alpha^{p} \mu\left(E_{\alpha} \cap B\right),
$$

where $0<p<\infty$, with $\mu$ replaced by $w d \mu$ and $p$ replaced by $p-1$, to get

$$
\begin{aligned}
\int_{E_{\alpha} \cap B} & w_{\beta}(x)^{p} d x \leq \int_{E_{\alpha} \cap B} w_{\beta}(x)^{p-1} w(x) d x \\
& =(p-1) \int_{\alpha}^{\infty} \lambda^{p-2} \int_{\left\{w_{\beta}>\lambda\right\} \cap B} w(x) d x d \lambda+\alpha^{p-1} \int_{E_{\alpha} \cap B} w(x) d x \\
& \leq c(p-1) \int_{\alpha}^{\infty} \lambda^{p-1}\left|\left\{w_{\beta}>\lambda\right\} \cap B\right| d \lambda+c \alpha^{p}\left|E_{\alpha} \cap B\right| .
\end{aligned}
$$

Next we estimate the first integral on the right side using (2.17) and find

$$
\int_{\alpha}^{\infty} \lambda^{p-1}\left|\left\{w_{\beta}>\lambda\right\}\right| d \lambda=\frac{1}{p}\left(\int_{E_{\alpha} \cap B} w_{\beta}(x)^{p} d x-\alpha^{p}\left|E_{\alpha} \cap B\right|\right) .
$$

Hence we obtain

$$
\int_{E_{\alpha} \cap B} w_{\beta}(x)^{p} d x \leq c \frac{p-1}{p} \int_{E_{\alpha} \cap B} w_{\beta}(x)^{p} d x+\frac{c}{p} \alpha^{p}\left|E_{\alpha} \cap B\right| .
$$

Choosing $p>1$ such that $c(p-1) / p<1$ and using the fact that all terms in the previous inequality are finite, we conclude

$$
\int_{E_{\alpha} \cap B} w_{\beta}(x)^{p} d x \leq \frac{c}{c-p(c-1)} \alpha^{p}\left|E_{\alpha} \cap B\right| .
$$

Finally, as $\beta \rightarrow \infty$, the monotone convergence theorem gives (2.16). This proves the lemma.

2.18. Remark. Both the bound for $p$ and the constant in (2.16) are the best possible as is easily seen by taking $B$ to be the unit ball and $w: \mathbf{R}^{n} \rightarrow[0, \infty], w(x)=|x|^{n(1 / c-1)}$. 


\section{Proof of Theorem 1.3}

Let $B$ be a ball in $\mathbf{R}^{n}$ and suppose that $w \in \mathcal{A}_{1}$ with the constant $c_{w}$. Using (2.5) we see that

$$
\int_{E_{\lambda} \cap B} w(x) d x \leq\left(c_{w}+\eta\left(c_{w}-1\right)\right) \lambda\left|E_{\lambda} \cap B\right|, \quad \underset{x \in B}{\operatorname{essinf}} w(x) \leq \lambda<\infty,
$$

where $\eta$ is the constant given by Lemma 2.4. This shows that $w$ fulfills the assumptions of Lemma 2.14 and from (2.16) we conclude that

$$
\begin{aligned}
\int_{B} w(x)^{p} d x & =\int_{B \backslash E_{\alpha}} w(x)^{p} d x+\int_{B \cap E_{\alpha}} w(x)^{p} d x \\
& \leq \alpha^{p}\left|B \backslash E_{\alpha}\right|+c \alpha^{p}\left|B \cap E_{\alpha}\right| \\
& \leq c \alpha^{p}|B|,
\end{aligned}
$$

whenever $\operatorname{essinf}_{x \in B} w(x) \leq \alpha<\infty$ and

$$
1 \leq p<1+\frac{1}{(\eta+1)\left(c_{w}-1\right)}
$$

In particular, we get

$$
\left(\frac{1}{|B|} \int_{B} w(x)^{p} d x\right)^{1 / p} \leq c \frac{1}{|B|} \int_{B} w(x) d x .
$$

The constant $c$ does not depend on $B$ and hence we may repeat the same reasoning in every ball $B$ and we see that $w$ satisfies the reverse Hölder inequality for every $p>1$ such that (1.4) holds if we take $\nu=(\eta+1)^{-1}$. This completes the proof of Theorem 1.3.

Acknowledgements. I would like to thank Michael Korey for making valuable comments on early versions of this paper.

\section{References}

1. B. BOJARSKI, Remarks on the stability of reverse Hölder inequalities and quasiconformal mappings, Ann. Acad. Sci. Fenn. Ser. A I Math. 10 (1985), 89-94.

2. B. Bojarski, C. Sbordone And I. WiK, The Muckenhoupt class $A_{1}(\mathbf{R})$, Studia Math. 101(2) (1992), 155-163. 
3. R. Coifman and C. Fefferman, Weighted norm inequalities for maximal functions and singular integrals, Studia Math. 51 (1974), 241-250.

4. D. Cruz-Uribe, SFO, And C. J. Neugebauer, The structure of the reverse Hölder classes, Trans. Amer. Math. Soc. 347 (1995), 2941-2960.

5. M. DE GuZmán, "Differentiation of Integrals in $\mathbf{R}^{n}$," Lecture Notes in Mathematics 481, Springer-Verlag, 1975.

6. F. W. GeHring, The $L^{p}$-integrability of the partial derivatives of a quasiconformal mapping, Acta Math. 130 (1973), 265-277.

7. J. García-Cuerva And J. L. Rubio De Francia, "Weighted Norm Inequalities and Related Topics," North Holland Math. Studies 116, North Holland, 1985.

8. Z. Füredi And P. A. LoeB, On the best constant for the Besicovitch covering Theorem, Proc. Amer. Math. Soc. 121 (1994), 1063-1073.

9. T. IWANIEC, On $L^{p}$-integrability in PDE's and quasiregular mappings for large exponents, Ann. Acad. Sci. Fenn. Ser. A I Math. 7 (1982), 301-322.

10. P. W. Jones, Factorization of $A_{p}$ weights, Ann. of Math. 111 (1980), 511-530.

11. J. Kinnunen, Sharp results on reverse Hölder inequalities, Ann. Acad. Sci. Fenn. Ser. A I Math. Dissertationes 95 (1994), 1-34.

12. A. A. KorenovskiI, The exact continuation of a reverse Hölder inequality and Muckenhoupt's conditions, Math. Notes 52 (1993), 1192-1201.

13. A. A. KorenovskiǏ, The reverse Hölder inequality, the Muckenhoupt condition, and equimeasurable rearrangements of functions, Russian Acad. Sci. Dokl. Math. 45 (1992), 301-304.

14. M. B. Korey, Ideal weights: doubling and absolute continuity with asymptotically optimal bounds, Preprint, Max-Planck-Institut für Mathematik, Bonn (1995).

15. L. Migliaccio, Some characterizations of Gehring $G_{p}$-class, Houston J. Math. 19 (1993), 89-95.

16. B. Muckenhoupt, Weighted norm inequalities for the Hardy maximal function, Trans. Amer. Math. Soc. 165 (1972), 207-226.

17. C. J. Neugebauer, The precise range of indices for the $R H_{r}$-and $A_{p}$-classes, Preprint (1996).

18. A. Politis, Sharp results on the relation between weight spaces and BMO, Ph.D. Thesis, University of Chicago (1995). 
19. Y. G. Reshetnyak, Stability estimates in Liouville's theorem, and the $L^{p}$-integrability of the derivatives of quasi-conformal mappings, Siberian Math. J. 17 (1976), 653-674.

20. I. WIK, Note on a theorem by Reshetnyak-Gurov, Studia Math. 86 (1987), 287-290.

21. I. WIK, Reverse Hölder inequalities with constant close to 1, Ricerche Mat. 39 (1990), 151-157.

\author{
Department of Mathematics \\ P.O.Box 4 \\ University of Helsinki \\ FIN-00014 \\ FINLAND
}

e-mail: Juha.Kinnunen@Helsinki.Fi

Primera versió rebuda el 17 de febrer de 1997, darrera versió rebuda el 20 de maig de 1997 